\title{
EFFECT OF COMPOST, SALICYLIC AND ASCORBIC ACIDS TREATMENTS ON CORM PRODUCTION AND CHEMICAL CONSTITUENTS OF GLADIOLUS GRANDIFLORUS CV. WHITE PROSPERITY
}

\begin{abstract}
M.A.H. Abdou; A.A. El-Sayed and A.R.M. Khalil
Horticulture Dept., Fac. Agric., Minia Univ., Egypt.

ABSTRACT: A field experiment was conducted to investigate the effect of plant compost $(0,7.5,10.0$ and 12.5 ton/fed) and salicylic and/or ascorbic acids (each at 0,100 or $200 \mathrm{ppm}$ ) on corm production and chemical constituents of Gladiolus grandiflorus cv. White Prosperity.

The obtained results indicated that corm diameter, number of cormels/plant, dry weight of corm and cormels, as well as, chemical constituents including chlorophyll $\mathrm{a}, \mathrm{b}$, carotenoids and percentages of $\mathrm{N}$, $\mathrm{P}$ and $\mathrm{K}$ in the corms were gradually increased by increasing the level of compost. Significant differences were detected among four used treatments. So, the high level of compost resulted the highest values.

All six treatments of salicylic acid and/or ascorbic acid significantly increased corm and cormels production and their content of chemical constituents in comparison of the control plants. The treatments of salicylic acid (100 ppm) plus ascorbic acid $(100 \mathrm{ppm})$ followed by ascorbic acid (200 ppm), then salicylic acid (200 ppm) were more effective than other treatments for corm and cormels production, as well

Scientific J. Flowers \& Ornamental Plants, 1(3):233-242 (2014).

Received:

$4 / 11 / 2014$

Revised by:

Prof. Dr. A.Z. Sarhan, Cairo Univ.

Prof. Dr. S.M. Shahin, Hort. Res. Inst., ARC.

as, the percentage of nitrogen and potassium without significant differences were detected between the first and second treatments in most cases. While the treatments of salicylic plus ascorbic acids, each at 100 ppm followed by salicylic acid at $200 \mathrm{ppm}$ were more effective for the pigments content (mg/g f.w.) and phosphorus percentage without significant differences between them.

The interaction between compost, salicylic acid and/or ascorbic acid was significant for all previous characters, except $\mathrm{K} \%$. The highest values of corm and cormels production were achieved by 12.5 ton/fed compost treatment in combination with salicylic acid $(100 \mathrm{ppm})+$ ascorbic acid (100 ppm) or ascorbic acid (200 ppm), while the interaction treatments of compost $(12.5 \mathrm{ton} / \mathrm{fed}) \times$ salicylic plus ascorbic acids, each at $100 \mathrm{ppm}$ or compost $(12.5 \mathrm{ton} / \mathrm{fed}) \times$ salicylic acid at $200 \mathrm{ppm}$ recorded the highest contents of pigments and elements of $\mathrm{N}$ and $\mathrm{P}$.
\end{abstract}

Key words: Gladiolus grandiflorus, cv. White Prosperity, compost, salicylic acid, ascorbic acid, corm production, chemical constituents.

\section{INTRODUCTION}

Gladiolus grandiflorus, L. plant is considered one of the most important flowering bulbs grown in Egypt. There are fast expands in areas planted with gladiolus in Egypt in order to meet the increase demand for gladiolus flowers for local market and exporting, Gladiolus plants propagated by corms and cormels.

Organic fertilization, salicylic acid and ascorbic acid are among the important agricultural treatments which have been proved to improve corm production of gladiolus plants. 
The effect of organic fertilization on increasing corm diameter, number of cormels and dry weight of corm and cormels of gladiolus were reported by many investigators, such as Gangadharan and Gopinth (2000), Zaghloul and Moghazy (2001), Atta-Alla et al. (2003), Ruppenthal and Castro (2005), Chandar et al. (2012), Ahmed (2013) and Abdou et al. (2013) who found that compost fertilizer improved the chemical composition of gladiolus plants.

Salicylic acid treatments were found to have stimulating effect on corm production and chemical composition of gladiolus such as those raveled by Sajjad et al. (2014) and Padmalatha et al. (2014).

In regard to corm and cormels production, as well as, chemical constituents of gladiolus, ascorbic acid treatments were found to increase corm diameter, number of cormels, dry weight of corm and cormels and pigments (chlorophyll a, b and carotenoids contents), as well as, NPK \% (Abdel Aziz et al., 2009 and Abo Leila and Eid, 2011).

The aim of this work was to study the effect of application compost fertilization and spraying with salicylic and ascorbic acids on corm and cormels production, as well as, chemical constituents of Gladiolus grandiflorus cv. White Prosperity.

\section{MATERIALS AND METHODS}

The present study was carried out at the Nursery of Ornamental Plants, Faculty of Agriculture, Minia University during two successive seasons of 2012/2013 and 2013/2014 on gladiolus plants.

Gladiolus grandiflorus cv. White Prosperity corms were obtained from Holland by Basiony nurseries, Cairo, Egypt. Average corm diameter was 2.8 and $3.1 \mathrm{~cm}$ and corm weight was 9.6 and $10.1 \mathrm{~g}$ for the first and second seasons, respectively. Corms were planted on October $1^{\text {st }}$ for both seasons in $1.5 \times 2.2 \mathrm{~m}$ plots containing 3 ridges, 50 $\mathrm{cm}$ apart. Corms were planted in hills, $20 \mathrm{~cm}$ apart (10 corms/ridge) at a depth of $5 \mathrm{~cm}$ under ground surface in clay loam soil. The physical and chemical analysis of the used soil are shown in Table (a).

The split plot design with three replicates was followed in this experiment. The four levels of compost fertilization treatments $(0.0,7.5,10.0$ and 12.5 ton/fed) were considered as main plots and the seven salicylic and/or ascorbic acids treatments (control, salicylic acid at 100 or 200 ppm, ascorbic acid at 100 or $200 \mathrm{ppm}$, salicylic acid + ascorbic acid, each at 100 or 200 ppm) were put in the sub-plots.

The compost was added before planting during the soil preparation. The chemical analysis of the compost is shown in Table (b).

Salicylic acid and/or ascorbic acid were applied, by hand sprayer, 3 times, one month and two months from planting date and after flowers cut for corm and cormels production. The plants were sprayed till run off. All agricultural practices were performed as usual in the region.

Table a. Physical and chemical properties of the used soil in both seasons.

\begin{tabular}{|c|c|c|c|c|c|c|}
\hline \multirow{2}{*}{ Soil Character } & \multicolumn{2}{|c|}{ Value } & \multirow{2}{*}{\multicolumn{2}{|c|}{ Soil Character }} & \multicolumn{2}{|c|}{ Value } \\
\hline & $2012 / 2013$ & $2013 / 2014$ & & & $2012 / 2013$ & 2013/2014 \\
\hline Sand $\%$ & 28.98 & 28.90 & \multicolumn{2}{|c|}{ Available P \% } & 15.67 & 15.58 \\
\hline Silt \% & 29.87 & 30.64 & \multicolumn{2}{|c|}{ Exch. $\mathrm{K}^{+} \mathrm{mg} / 100 \mathrm{~g}$} & 2.85 & 2.64 \\
\hline Clay \% & 41.15 & 40.46 & \multicolumn{2}{|c|}{ Exch. $\mathrm{Ca}^{++} \mathrm{mg} / 100 \mathrm{~g}$} & 31.12 & 31.43 \\
\hline Soil type & Clay loam & Clay loam & \multicolumn{2}{|c|}{ Exch. $\mathrm{Na}^{+} \mathrm{mg} / 100 \mathrm{~g}$} & 2.51 & 2.50 \\
\hline Organic matter \% & 1.54 & 1.59 & \multirow{5}{*}{$\begin{array}{c}\text { DTPA } \\
\text { Ext. ppm }\end{array}$} & $\mathrm{Fe}$ & 8.23 & 8.11 \\
\hline $\mathrm{Ca} \mathrm{CO}_{3} \%$ & 2.11 & 2.10 & & $\mathrm{Cu}$ & 2.01 & 2.00 \\
\hline pH (1: 2.5) & 7.75 & 7.71 & & $\mathbf{Z n}$ & 2.87 & 2.89 \\
\hline E. C. (m mhos/cm) & 1.08 & 1.06 & & Mn & 8.11 & 8.15 \\
\hline Total N \% & 0.08 & 0.06 & & & & \\
\hline
\end{tabular}


Table b. Chemical analysis of the compost used in the two seasons.

\begin{tabular}{lclc}
\hline Properties & Value & Properties & Value \\
\hline Organic carbon & 25.1 & Total P (\%) & 0.5 \\
Humidity (\%) & 25 & Total K (\%) & 1.0 \\
Organic matter (\%) & 44 & Fe (ppm) & 1750 \\
C/N ratio & 17.5 & Zn (ppm) & 60 \\
pH (1:2.5) & 8.0 & Mn (ppm) & 125 \\
E.C. (m. mhos/cm) & 5 & Cu (ppm) & 200 \\
Total N (\%) & 1.5 & & \\
\hline
\end{tabular}

\section{The following data were recorded:}

1- Under ground parts characters at harvesting after the foliage had dried (the under ground parts were lifted 2 months after cut spikes): corm diameter (cm), number of new cormels/plant and dry weights of corm and cormels $(\mathrm{g})$.

2- Determination of some chemical constituents: leaf samples were taken after 75 days from planting to determine chlorophyll $\mathrm{a}, \mathrm{b}$ and carotenoids as $\mathrm{mg} / \mathrm{g}$ f.w. using the method described by Moran (1982). The percentages of N, P and $\mathrm{K}$ in the dry corms (samples were taken after two months from flowering ending) were estimated according to the methods described by Wilde et al. (1985), Champan and Pratt (1975) and Cottenie et al. (1982), respectively.

All obtained data were tabulated and statistically analyzed according to MSTATC (1986) and the L.S.D. test at $5 \%$ was followed to compare between the means.

\section{RESULTS AND DISCUSSION}

\section{1- Corm and cormels production:}

The data listed in Tables (1 and 2) during both seasons indicated that corm diameter, corm dry weight, number of cormels/plant and dry weight of cormels/plant were significantly increased with increasing compost level in comparison with untreated control plants. Among the three compost treatments, the high level treatment (12.5 ton/fed) resulted the highest values for all corm and cormels production over both low and medium compost treatments in the two seasons. Similar results were also revealed on gladiolus plants by Gangadharan and Gopinath (2000), Zaghloul and Moghazy (2001), Atta-Alla et al. (2003), Ruppenthal and Castro (2005), Chandar et al. (2012) and Ahmed (2013).

The increase in the corm and cormels production was attributed to the positive effect of organic fertilizers on improving the vegetative growth, as well as, stimulating the photosynthetic pigments (Table, 3) which reflected on increasing the under ground parts of gladiolus.

In relation to salicylic and/or ascorbic acids treatments, the six treatments suppressed, significantly at 5\% level, the control treatment in both first and second seasons in producing wider corm, higher new cormels/plant and heavier dry weights of corms and cormels as shown in Tables (1 and 2). Among the six treatments, the combined one at low concentration (salicylic acid plus ascorbic acid, each at $100 \mathrm{ppm}$ ) followed by ascorbic acid (200 ppm), then salicylic acid (200 ppm) gave the highest values for corm and cormels production. Similar observations were pointed out on gladiolus plants such as Sajjad et al. (2014) and Padmalatha et al. (2014) regarding the effects of salicylic acid. While, Abdel Aziz et al. (2009) and Abo Leila and Eid, 2011 concerning the effects of ascorbic acid on gladiolus corm production.

The stimulatory effect of salicylic acid and/or ascorbic acid treatments on corm and cormels production may be due to their mode of action, nutrient uptake, antioxidant defense and regulation of photosynthesis and growth. Consequently increase in all corm production (Khan et al., 2003 and Blokhina et al., 2003).

The interaction between compost, salicylic and/or ascorbic acids treatments was significant for corm diameter, number of cormels/plant and dry weights of corm and cormels per plant in the two seasons (Tables 1 and 2). The highest values were obtained 
Table 1. Effect of compost, salicylic and ascorbic acid treatments on corm diameter and corm dry weight of Gladiolus grandiflorus cv. White Prosperity plants during 2012/2013 and 2013/2014 seasons.

\begin{tabular}{|c|c|c|c|c|c|c|c|c|c|c|}
\hline \multirow{3}{*}{$\begin{array}{c}\text { Salicylic and } \\
\text { ascorbic acids } \\
\text { treatments (B) }\end{array}$} & \multicolumn{10}{|c|}{ Compost levels (ton/feddan) (A) } \\
\hline & \multicolumn{5}{|c|}{$1^{\text {st }}$ Season } & \multicolumn{5}{|c|}{$2^{\text {nd }}$ Season } \\
\hline & $\mathbf{0}$ & 7.5 & 10.0 & 12.5 & Mean (B) & $\mathbf{0}$ & 7.5 & 10.0 & 12.5 & Mean (B) \\
\hline & \multicolumn{10}{|c|}{ Corm diameter $(\mathrm{cm})$} \\
\hline Control & 3.15 & 3.68 & 3.99 & 4.34 & 3.79 & 3.26 & 3.88 & 4.18 & 4.55 & 3.97 \\
\hline Sal. at 100 ppm & 3.42 & 3.95 & 4.27 & 4.65 & 4.07 & 3.53 & 4.26 & 4.58 & 4.96 & 4.24 \\
\hline Sal. at 200 ppm & 3.99 & 4.55 & 4.89 & 4.91 & 4.59 & 4.05 & 4.67 & 4.88 & 5.26 & 4.72 \\
\hline Asc. at 100 ppm & 3.53 & 4.08 & 4.39 & 4.70 & 4.18 & 3.64 & 4.37 & 4.59 & 5.08 & 4.42 \\
\hline Asc. at 200 ppm & 4.02 & 4.78 & 4.89 & 5.02 & 4.68 & 4.15 & 4.96 & 5.17 & 5.35 & 4.91 \\
\hline (Sal.+Asc.) at $100 \mathrm{ppm}$ & 4.06 & 4.86 & 4.95 & 5.16 & 4.76 & 4.30 & 5.18 & 5.26 & 5.63 & 5.09 \\
\hline (Sal.+Asc.) at $200 \mathrm{ppm}$ & 3.59 & 4.26 & 4.58 & 4.91 & 4.34 & 3.73 & 4.46 & 4.67 & 5.16 & 4.51 \\
\hline Mean (A) & 3.68 & 4.30 & 4.57 & 4.81 & & 3.81 & 4.54 & 4.76 & 5.16 & \\
\hline \multirow[t]{2}{*}{ L.S.D. at 5\% } & \multicolumn{2}{|c|}{ A: 0.21} & B: 0.10 & \multicolumn{2}{|c|}{ AB: 0.20} & \multicolumn{2}{|c|}{ A: 0.32} & B: 0.18 & \multicolumn{2}{|c|}{ AB: 0.36} \\
\hline & \multicolumn{10}{|c|}{ Corm dry weight (g) } \\
\hline Control & 10.55 & 14.70 & 15.93 & 17.06 & 14.56 & 12.99 & 15.46 & 16.65 & 18.18 & 15.82 \\
\hline Sal. at 100 ppm & 14.50 & 15.73 & 18.22 & 18.51 & 16.74 & 14.05 & 16.93 & 18.16 & 19.74 & 17.22 \\
\hline Sal. at 200 ppm & 15.84 & 18.46 & 19.52 & 19.52 & 18.34 & 15.62 & 18.52 & 19.35 & 20.32 & 18.45 \\
\hline Asc. at $100 \mathrm{ppm}$ & 14.04 & 16.24 & 18.11 & 18.69 & 16.77 & 14.50 & 17.38 & 18.20 & 20.32 & 17.60 \\
\hline Asc. at 200 ppm & 15.92 & 18.94 & 19.39 & 19.90 & 18.54 & 16.44 & 19.64 & 20.48 & 21.86 & 19.61 \\
\hline (Sal.+Asc.) at $100 \mathrm{ppm}$ & 16.08 & 19.06 & 19.61 & 20.65 & 18.85 & 17.05 & 20.50 & 20.86 & 22.31 & 20.18 \\
\hline (Sal.+Asc.) at $200 \mathrm{ppm}$ & 14.28 & 16.95 & 18.22 & 19.54 & 17.25 & 14.85 & 17.76 & 18.57 & 20.34 & 17.88 \\
\hline Mean (A) & 14.46 & 17.15 & 18.43 & 19.12 & & 15.07 & 18.01 & 18.90 & 20.44 & \\
\hline L.S.D. at 5\% & \multicolumn{2}{|c|}{ A: 0.68} & B: 0.51 & \multicolumn{2}{|c|}{ AB: 1.02} & \multicolumn{2}{|c|}{ A: 1.54} & B: 0.58 & \multicolumn{2}{|c|}{ AB: 1.16} \\
\hline
\end{tabular}

Sal.: Salicylic acid

Asc.: Ascorbic acid

Table 2. Effect of compost, salicylic and ascorbic acid treatments on number of cormels/plant and cormels dry weight of Gladiolus grandiflorus cv. White Prosperity plants during 2012/2013 and 2013/2014 seasons.

\begin{tabular}{|c|c|c|c|c|c|c|c|c|c|c|}
\hline \multirow{3}{*}{$\begin{array}{c}\text { Salicylic and } \\
\text { ascorbic acids } \\
\text { treatments (B) }\end{array}$} & \multicolumn{10}{|c|}{ Compost levels (ton/feddan) (A) } \\
\hline & \multicolumn{5}{|c|}{$1^{\text {st }}$ Season } & \multicolumn{5}{|c|}{$2^{\text {nd }}$ Season } \\
\hline & $\mathbf{0}$ & 7.5 & $\mathbf{1 0 . 0}$ & 12.5 & Mean (B) & $\mathbf{0}$ & 7.5 & 10.0 & 12.5 & Mean (B) \\
\hline & \multicolumn{10}{|c|}{ Number of cormels/plant } \\
\hline Control & 30.2 & 35.7 & 39.8 & 43.9 & 29.9 & 33.1 & 39.8 & 44.6 & 53.8 & 42.8 \\
\hline Sal. at 100 ppm & 33.3 & 38.9 & 43.1 & 47.3 & 40.7 & 37.2 & 43.7 & 48.3 & 57.8 & 46.8 \\
\hline Sal. at 200 ppm & 40.1 & 46.1 & 51.0 & 54.9 & 48.0 & 41.4 & 48.0 & 52.9 & 62.5 & 51.2 \\
\hline Asc. at 100 ppm & 35.4 & 41.1 & 45.2 & 49.6 & 42.8 & 39.3 & 45.8 & 50.5 & 59.9 & 48.9 \\
\hline Asc. at 200 ppm & 41.6 & 47.8 & 51.2 & 55.8 & 49.1 & 42.5 & 49.2 & 54.2 & 63.8 & 52.4 \\
\hline (Sal.+Asc.) at 100 ppm & 42.2 & 48.4 & 51.8 & 56.4 & 49.7 & 42.9 & 49.8 & 54.9 & 64.6 & 53.05 \\
\hline (Sal.+Asc.) at $200 \mathrm{ppm}$ & 37.1 & 43.0 & 47.8 & 51.6 & 44.9 & 40.8 & 47.3 & 52.1 & 60.5 & 50.2 \\
\hline Mean (A) & 37.0 & 43.0 & 47.1 & 51.4 & & 39.6 & 46.2 & 51.1 & 59.0 & \\
\hline \multirow[t]{2}{*}{ L.S.D. at 5\% } & \multicolumn{2}{|c|}{ A: 3.0} & B: 1.7 & \multicolumn{2}{|c|}{ AB: 3.4} & \multicolumn{2}{|c|}{ A: 2.8} & B: 1.9 & \multicolumn{2}{|c|}{ AB: 3.8} \\
\hline & \multicolumn{10}{|c|}{ Cormels dry weight (g) } \\
\hline Control & 10.06 & 11.91 & 13.28 & 14.65 & 12.48 & 11.17 & 13.43 & 15.06 & 18.15 & 14.45 \\
\hline Sal. at 100 ppm & 10.83 & 12.60 & 13.97 & 15.34 & 13.19 & 12.35 & 14.51 & 16.03 & 19.21 & 15.53 \\
\hline Sal. at 200 ppm & 12.96 & 14.89 & 16.51 & 17.76 & 15.53 & 14.04 & 16.03 & 17.64 & 20.59 & 17.08 \\
\hline Asc. at 100 ppm & 11.52 & 13.39 & 14.69 & 16.14 & 13.94 & 13.07 & 15.20 & 16.86 & 19.89 & 16.26 \\
\hline Asc. at 200 ppm & 13.46 & 15.51 & 16.59 & 18.10 & 15.92 & 14.04 & 16.26 & 17.93 & 21.29 & 17.38 \\
\hline (Sal.+Asc.) at $100 \mathrm{ppm}$ & 13.67 & 15.73 & 16.81 & 18.36 & 16.14 & 14.18 & 16.45 & 18.17 & 21.64 & 17.61 \\
\hline (Sal.+Asc.) at $200 \mathrm{ppm}$ & 12.13 & 14.05 & 15.61 & 16.87 & 14.67 & 13.72 & 15.89 & 17.56 & 20.38 & 16.89 \\
\hline Mean (A) & 12.09 & 14.01 & 15.35 & 16.75 & & 13.23 & 15.40 & 17.04 & 20.16 & \\
\hline L.S.D. at $5 \%$ & \multicolumn{2}{|c|}{ A: 1.32} & B: 0.64 & \multicolumn{2}{|c|}{ AB: 1.28} & \multicolumn{2}{|c|}{ A: 1.28} & B: 0.53 & \multicolumn{2}{|c|}{ AB: 1.06} \\
\hline
\end{tabular}

Sal.: Salicylic acid Asc.: Ascorbic acid 
Table 3. Effect of compost, salicylic and ascorbic acid treatments on chlorophyll a, b and carotenoids of Gladiolus grandiflorus cv. White Prosperity plants during 2012/2013 and 2013/2014 seasons.

\begin{tabular}{|c|c|c|c|c|c|c|c|c|c|c|}
\hline \multirow{3}{*}{$\begin{array}{c}\text { Salicylic and } \\
\text { ascorbic acids } \\
\text { treatments (B) }\end{array}$} & \multicolumn{10}{|c|}{ Compost levels (ton/feddan) (A) } \\
\hline & \multicolumn{5}{|c|}{$1^{\text {st }}$ Season } & \multicolumn{5}{|c|}{$2^{\text {nd }}$ Season } \\
\hline & $\mathbf{0}$ & 7.5 & 10.0 & 12.5 & Mean (B) & $\mathbf{0}$ & 7.5 & 10.0 & 12.5 & Mean (B) \\
\hline & \multicolumn{10}{|c|}{ Chlorophyll a (mg/g f.w.) } \\
\hline Control & 2.328 & 2.369 & 2.408 & 2.445 & 2.388 & 2.337 & 2.378 & 2.419 & 2.456 & 2.398 \\
\hline Sal. at 100 ppm & 2.365 & 2.406 & 2.447 & 2.488 & 2.427 & 2.374 & 2.415 & 2.456 & 2.497 & 2.436 \\
\hline Sal. at 200 ppm & 2.500 & 2.536 & 2.565 & 2.598 & 2.550 & 2.515 & 2.553 & 2.580 & 2.613 & 2.565 \\
\hline Asc. at 100 ppm & 2.396 & 2.428 & 2.459 & 2.489 & 2.443 & 2.405 & 2.437 & 2.468 & 2.499 & 2.452 \\
\hline Asc. at 200 ppm & 2.469 & 2.508 & 2.538 & 2.567 & 2.521 & 2.481 & 2.520 & 2.551 & 2.579 & 2.533 \\
\hline (Sal.+Asc.) at $100 \mathrm{ppm}$ & 2.526 & 2.558 & 2.586 & 2.616 & 2.572 & 2.541 & 2.573 & 2.601 & 2.636 & 2.588 \\
\hline (Sal.+Asc.) at 200 ppm & 2.437 & 2.469 & 2.499 & 2.529 & 2.484 & 2.448 & 2.480 & 2.510 & 2.540 & 2.495 \\
\hline Mean (A) & 2.432 & 2.468 & 2.500 & 2.533 & & 2.443 & 2.479 & 2.512 & 2.546 & \\
\hline \multirow[t]{2}{*}{ L.S.D. at $5 \%$} & \multicolumn{2}{|c|}{ A: 0.031} & B: 0.025 & \multicolumn{2}{|c|}{ AB: 0.050} & \multicolumn{2}{|c|}{ A: 0.032} & B: 0.024 & \multicolumn{2}{|c|}{ AB: 0.048} \\
\hline & \multicolumn{10}{|c|}{ Chlorophyll b (mg/g f.w.) } \\
\hline Control & 0.756 & 0.769 & 0.783 & 0.796 & 0.776 & 0.759 & 0.773 & 0.787 & 0.799 & 0.780 \\
\hline Sal. at 100 ppm & 0.768 & 0.782 & 0.796 & 0.809 & 0.789 & 0.772 & 0.786 & 0.801 & 0.814 & 0.793 \\
\hline Sal. at 200 ppm & 0.813 & 0.825 & 0.835 & 0.847 & 0.830 & 0.820 & 0.833 & 0.845 & 0.856 & 0.839 \\
\hline Asc. at 100 ppm & 0.779 & 0.788 & 0.799 & 0.810 & 0.794 & 0.782 & 0.791 & 0.803 & 0.814 & 0.798 \\
\hline Asc. at 200 ppm & 0.803 & 0.816 & 0.826 & 0.836 & 0.820 & 0.808 & 0.821 & 0.832 & 0.843 & 0.826 \\
\hline (Sal.+Asc.) at 100 ppm & 0.822 & 0.833 & 0.842 & 0.852 & 0.837 & 0.830 & 0.843 & 0.854 & 0.867 & 0.849 \\
\hline (Sal.+Asc.) at 200 ppm & 0.792 & 0.803 & 0.813 & 0.823 & 0.808 & 0.795 & 0.806 & 0.814 & 0.828 & 0.811 \\
\hline Mean (A) & 0.790 & 0.802 & 0.813 & 0.825 & & 0.795 & 0.808 & 0.819 & 0.832 & \\
\hline \multirow[t]{2}{*}{ L.S.D. at $5 \%$} & \multicolumn{2}{|c|}{ A: 0.010} & B: 0.008 & \multicolumn{2}{|c|}{ AB: 0.016} & \multicolumn{2}{|c|}{ A: 0.011} & B: 0.011 & \multicolumn{2}{|c|}{ AB: 0.022} \\
\hline & \multicolumn{10}{|c|}{ Carotenoids (mg/g f.w.) } \\
\hline Control & 0.798 & 0.808 & 0.823 & 0.835 & 0.816 & 0.802 & 0.815 & 0.830 & 0.841 & 0.822 \\
\hline Sal. at 100 ppm & 0.809 & 0.823 & 0.835 & 0.848 & 0.829 & 0.817 & 0.831 & 0.843 & 0.856 & 0.837 \\
\hline Sal. at 200 ppm & 0.853 & 0.865 & 0.876 & 0.889 & 0.871 & 0.862 & 0.874 & 0.885 & 0.898 & 0.880 \\
\hline Asc. at 100 ppm & 0.818 & 0.829 & 0.840 & 0.850 & 0.834 & 0.827 & 0.838 & 0.849 & 0.860 & 0.844 \\
\hline Asc. at 200 ppm & 0.843 & 0.857 & 0.867 & 0.878 & 0.861 & 0.851 & 0.869 & 0.879 & 0.886 & 0.871 \\
\hline (Sal.+Asc.) at $100 \mathrm{ppm}$ & 0.863 & 0.874 & 0.883 & 0.894 & 0.879 & 0.871 & 0.885 & 0.896 & 0.909 & 0.889 \\
\hline (Sal.+Asc.) at 200 ppm & 0.833 & 0.845 & 0.853 & 0.865 & 0.849 & 0.839 & 0.854 & 0.863 & 0.874 & 0.858 \\
\hline Mean (A) & 0.831 & 0.843 & 0.854 & 0.866 & & 0.838 & 0.852 & 0.864 & 0.875 & \\
\hline L.S.D. at 5\% & \multicolumn{2}{|c|}{ A: 0.009} & B: 0.008 & \multicolumn{2}{|c|}{ AB: 0.016} & \multicolumn{2}{|c|}{ A: 0.008} & B: 0.010 & \multicolumn{2}{|c|}{ AB: 0.020} \\
\hline
\end{tabular}


for all corm and cormels production parameters when gladiolus plants received compost at 12.5 ton/fed and sprayed with salicylic acid (100 ppm) + ascorbic acid (100 ppm) followed by sprayed with ascorbic acid (200 ppm), then salicylic acid (200 ppm).

\section{2- Chemical constituents:}

\section{a. Photosynthetic pigments:}

The contents of chlorophyll a, b and carotenoids in the fresh leaves of Gladiolus cv. White Prosperity were significantly promoted due to compost treatments, in the two growing seasons, in comparison with those of untreated plants as shown in Table (3). The high level of compost (12.5 ton/fed) gave the highest values for the three photosynthetic pigments in both seasons. This results may be attributed to the increase in nutrient elements and/or positive role of organic compost on the physical and chemical properties of the soil, that reflected on the growth and the pigments content. In harmony with these results regarding organic fertilization treatments were those reported by Abdou et al. (2013) on gladiolus, Bahadoran et al. (2011) on tuberose and Rajaei and Onsinejad (2014) on tulip cv. Bright Parrot.

In relation to the influence of salicylic and/or ascorbic acids treatments, chlorophyll $\mathrm{a}, \mathrm{b}$ and carotenoids contents were promoted, in the two seasons (Table, 3). Using both salicylic and ascorbic acids together each at low concentration was effective than the use of either one at low concentration or together each at $200 \mathrm{ppm}$. Also, the differences between any treatment and control was statistically significant, among the six treatments, salicylic acid (100 ppm) + ascorbic acid (100 ppm) or salicylic acid (200 ppm) resulted the highest values over all other treatments. This results may be attributed not only to the increase in nutrient elements, but also to the role of salicylic acid on stomatal regulation and photosynthesis and growth (Arfan et al., 2007).

Effect of the interactions between compost, salicylic acid and/or ascorbic acid treatments was significant in both seasons, for corm diameter, number of cormels/plant and dry weights of corm and cormels with the highest values being obtained due to the use of compost at 12.5 ton/fed in combination with salicylic acid (100 ppm) + ascorbic acid (100 ppm) or salicylic acid (200 ppm) as shown in Table (3).

\section{b. Corms content of nitrogen, phosphorus and potassium as percentages:}

In both seasons, increasing the level of compost linearly increased the percentages of $\mathrm{N}, \mathrm{P}$ and $\mathrm{K}$ in the corms. In this concern, the treatment with high level of compost (12.5 ton/fed) gave the highest percentages (Table, 4). On the other hand, the lowest values of $\mathrm{N}, \mathrm{P}$ and $\mathrm{K}$ in the corms of gladiolus were recorded by the plants treated with organic fertilization. Moreover, significant differences were detected between compost treatments and control one, also between compost treatments in all cases.

The results mentioned above, could be attributed to that application of compost improved soil properties, increase nutrients in area of roots, which increase nutrients uptake which in turn reflects on the corm quality.

These results are in agreement with those obtained by Abdou et al. (2013) and Sönmez et al. (2013) on gladiolus, Abd ElKarim (2001) on tuberose, Eliwa et al. (2009) on Iris tingitana cv. Wedegewood and El-Sayed et al. (2012) on Freesia reflecta.

Corms percentages of $\mathrm{N}, \mathrm{P}$ and $\mathrm{K}$ were significantly increased, in both seasons, as a result of spraying gladiolus with salicylic and/or ascorbic acids treatments in comparison with the control (Table, 4). The treatment of salicylic acid plus ascorbic acid, each at $100 \mathrm{ppm}$ followed by ascorbic acid (200 ppm), then salicylic acid (200 ppm) recorded the highest values for $\mathrm{N}$ and $\mathrm{K} \%$ in both seasons, without significant differences, except in case of $\mathrm{N} \%$ in the first season. The highest $\mathrm{P} \%$ was obtained with the treatments of salicylic acid plus ascorbic 
Table 4. Effect of compost, salicylic and ascorbic acid treatments on the percentages of nitrogen, phosphorus and potassium of Gladiolus grandiflorus cv. White Prosperity plants during 2012/2013 and 2013/2014 seasons.

\begin{tabular}{|c|c|c|c|c|c|c|c|c|c|c|}
\hline \multirow{3}{*}{$\begin{array}{c}\text { Salicylic and } \\
\text { ascorbic acids } \\
\text { treatments (B) }\end{array}$} & \multicolumn{10}{|c|}{ Compost levels (ton/feddan) (A) } \\
\hline & \multicolumn{5}{|c|}{$1^{\text {st }}$ Season } & \multicolumn{5}{|c|}{$2^{\text {nd }}$ Season } \\
\hline & $\mathbf{0}$ & 7.5 & 10.0 & 12.5 & Mean (B) & $\mathbf{0}$ & 7.5 & 10.0 & 12.5 & Mean (B) \\
\hline & \multicolumn{10}{|c|}{$\mathbf{N} \%$} \\
\hline Control & 1.642 & 1.893 & 2.104 & 2.216 & 1.964 & 1.717 & 1.966 & 2.177 & 2.289 & 2.037 \\
\hline Sal. at 100 ppm & 1.714 & 1.954 & 2.164 & 2.275 & 2.027 & 1.781 & 2.033 & 2.244 & 2.356 & 2.104 \\
\hline Sal. at 200 ppm & 1.807 & 2.090 & 2.282 & 2.390 & 2.132 & 1.873 & 2.157 & 2.347 & 2.534 & 2.228 \\
\hline Asc. at 100 ppm & 1.773 & 2.046 & 2.248 & 2.365 & 2.108 & 1.825 & 2.083 & 2.281 & 2.479 & 2.167 \\
\hline Asc. at 200 ppm & 1.819 & 2.112 & 2.294 & 2.393 & 2.155 & 1.884 & 2.168 & 2.358 & 2.545 & 2.239 \\
\hline (Sal.+Asc.) at $100 \mathrm{ppm}$ & 1.839 & 2.132 & 2.314 & 2.413 & 2.175 & 1.899 & 2.173 & 2.373 & 2.560 & 2.251 \\
\hline (Sal.+Asc.) at $200 \mathrm{ppm}$ & 1.745 & 1.995 & 2.216 & 2.318 & 2.060 & 1.849 & 2.123 & 2.320 & 2.513 & 2.201 \\
\hline Mean (A) & 1.763 & 2.032 & 2.232 & 2.339 & & 1.833 & 2.100 & 2.300 & 2.468 & \\
\hline \multirow[t]{2}{*}{ L.S.D. at $5 \%$} & \multicolumn{2}{|c|}{ A: 0.061} & B: 0.026 & \multicolumn{2}{|c|}{ AB: 0.052} & \multicolumn{2}{|c|}{ A: 0.078} & B: 0.024 & \multicolumn{2}{|c|}{ AB: 0.048} \\
\hline & \multicolumn{10}{|c|}{ P \% } \\
\hline Control & 0.219 & 0.292 & 0.317 & 0.328 & 0.289 & 0.224 & 0.298 & 0.324 & 0.338 & 0.296 \\
\hline Sal. at 100 ppm & 0.231 & 0.301 & 0.335 & 0.346 & 0.303 & 0.235 & 0.306 & 0.341 & 0.353 & 0.309 \\
\hline Sal. at 200 ppm & 0.290 & 0.361 & 0.386 & 0.399 & 0.359 & 0.298 & 0.369 & 0.395 & 0.410 & 0.368 \\
\hline Asc. at 100 ppm & 0.249 & 0.319 & 0.354 & 0.365 & 0.322 & 0.255 & 0.326 & 0.362 & 0.374 & 0.329 \\
\hline Asc. at 200 ppm & 0.287 & 0.356 & 0.381 & 0.401 & 0.356 & 0.295 & 0.364 & 0.390 & 0.412 & 0.365 \\
\hline (Sal.+Asc.) at $100 \mathrm{ppm}$ & 0.298 & 0.370 & 0.395 & 0.406 & 0.367 & 0.306 & 0.379 & 0.405 & 0.418 & 0.377 \\
\hline (Sal.+Asc.) at 200 ppm & 0.264 & 0.335 & 0.360 & 0.371 & 0.333 & 0.269 & 0.341 & 0.367 & 0.379 & 0.339 \\
\hline Mean (A) & 0.263 & 0.333 & 0.361 & 0.374 & & 0.269 & 0.340 & 0.369 & 0.383 & \\
\hline \multirow[t]{2}{*}{ L.S.D. at 5\% } & \multicolumn{2}{|c|}{ A: 0.010} & B: 0.012 & \multicolumn{2}{|c|}{ AB: 0.024} & \multicolumn{2}{|c|}{ A: 0.012} & B: 0.012 & \multicolumn{2}{|c|}{ AB: 0.024} \\
\hline & \multicolumn{10}{|c|}{ K\% } \\
\hline Control & 1.542 & 1.560 & 1.576 & 1.587 & 1.596 & 1.567 & 1.589 & 1.607 & 1.621 & 1.596 \\
\hline Sal. at 100 ppm & 1.551 & 1.569 & 1.585 & 1.596 & 1.605 & 1.578 & 1.600 & 1.618 & 1.632 & 1.607 \\
\hline Sal. at 200 ppm & 1.603 & 1.631 & 1.647 & 1.658 & 1.667 & 1.642 & 1.664 & 1.682 & 1.696 & 1.671 \\
\hline Asc. at 100 ppm & 1.562 & 1.580 & 1.596 & 1.607 & 1.616 & 1.590 & 1.612 & 1.630 & 1.644 & 1.619 \\
\hline Asc. at 200 ppm & 1.608 & 1.636 & 1.652 & 1.663 & 1.672 & 1.648 & 1.670 & 1.688 & 1.702 & 1.677 \\
\hline (Sal.+Asc.) at $100 \mathrm{ppm}$ & 1.612 & 1.640 & 1.656 & 1.667 & 1.676 & 1.655 & 1.677 & 1.695 & 1.709 & 1.684 \\
\hline (Sal.+Asc.) at $200 \mathrm{ppm}$ & 1.582 & 1.610 & 1.626 & 1.637 & 1.646 & 1.621 & 1.643 & 1.661 & 1.675 & 1.650 \\
\hline Mean (A) & 1.580 & 1.604 & 1.620 & 1.631 & & 1.614 & 1.635 & 1.654 & 1.668 & \\
\hline L.S.D. at 5\% & \multicolumn{2}{|c|}{ A: 0.006} & B: 0.009 & \multicolumn{2}{|c|}{ AB: N.S. } & \multicolumn{2}{|c|}{ A: 0.011} & B: 0.013 & \multicolumn{2}{|c|}{ AB: N.S. } \\
\hline
\end{tabular}


acid, each at $100 \mathrm{ppm}$ followed by salicylic acid (200 ppm), then ascorbic acid (200 ppm) without significant differences were detected between such three superior treatments. Salicylic acid has a role in nutrient uptake (Khan et al., 2003). Also, ascorbic acid is involved in a wide range of important functions as antioxidant defense and regulation of photosynthesis and growth (Blokhina et al., 2003). These positive effect of the used treatments led to promoted nutrient uptake and finally reflexes on the corms percentages of $\mathrm{N}, \mathrm{P}$ and $\mathrm{K}$.

Abdel Aziz et al. (2009) and Abo Leila and Eid (2011) on gladiolus found that ascorbic acid treatment increased NPK \%. Moreover, Sajjad et al. (2014) concluded that salicylic acid treatments $(0.1,0.4,0.7$ and $1.0 \mathrm{mM}$ ) increased $\mathrm{N}, \mathrm{P}$ and $\mathrm{K} \%$ in gladiolus.

Effect of the interaction treatments was significant, in both seasons, for $\mathrm{N}$ and $\mathrm{P} \%$ only. The highest values were obtained with the interaction treatments of 12.5 ton/fed compost $\times$ salicylic acid $(100 \mathrm{ppm})+$ ascorbic acid (100 ppm) followed by 12.5 ton/fed compost in combination with ascorbic acid (200 ppm) or salicylic acid (200 ppm) as shown in Table (4).

\section{REFERENCES}

Abdel Aziz, G.N.; Taha, L. and Ibrahim, S.M.M. (2009). Some studies on the effect of putrescine, ascorbic acid and thiamine on growth, flowering and some chemical constituents of gladiolus at Nubaria. Ozean J. of Applied Sci., 2(2):169-179.

Abd El-Karim, F.M. (2001). Studies on Some Factors Affecting Growth, Flowering, and Bulb Productivity of Polianthus tuberosa, L. Plant. Ph.D. Thesis, Fac. Agric., Kafr El-Sheikh, Tanta Univ.

Abdou, M.A.H.; Aly, M.K. and Ahmed, A.S.A. (2013). Effect of compost, biofertilization and some vitamins addition on Gladiolus grandiflorus. J.
Plant Production, Mansoura Univ., 4(12):1751-1761.

Abo Leila, B. and Eid, R. (2011). Improving gladiolus growth, flower keeping quality by using some vitamins application. Journal of American Science, 7(3):169174.

Ahmed, A.S.A. (2013). Physiological Studies on Gladiolus Plant. M.Sc. Thesis, Fac. Agric. Minia Univ. Egypt.

Arfan, M.; Athar, H.R. and Ashraf, M. (2007). Does exogenous application of salicylic acid through the rooting medium modulate growth and photosynthetic capacity in two differently adapted spring wheat cultivars under salt stress. J. Plant Physiol., 6:685-694.

Atta-Alla, H.K.; Zaghloul, M.A. and Hashish, K.H. (2003). Effect of organic manure and NPK fertilizers on vegetative growth, flowering and chemical composition of some Gladiolus cultivars. Ann. Agric. Sci., Moshtohor, 41(2):680691.

Bahadoran, M.; Salehi, H. and Eshghi, S. (2011). Growth and flowering of tuberose as affected by adding poultry litter to culture medium. Span. J. Agric. Res., 9(2):531-536.

Blokhina, O.; Virdainen, E. and Fagersted, K.V. (2003). Antioxidant, oxidative damage and oxygen deprivations stress. A Review Ann. Boi., 91:179-194.

Champan, H.D. and Pratt, P.F. (1975). Methods of Analysis for Soil, Plant and Water. Calif. Univ. Division of Agric. Sci., 172-174.

Chandar, I.; Rawat, I.; Lakhawat, S.S. and Yadav, K.K. (2012). Effect of organic manures and biofertilizers on the yield parameters of Gladiolus cv. White Prosperity. Ecol., Environ. and Conservation Paper, 18(1):91-94.

Cottenie, A.; Verloo, M.; Velghe, M. and Camerlynck, R. (1982). Chemical Analysis of Plant and Soil. Laboratory of 
Analytical and Agro Chemistry. State Univ., Ghent, Belgium.

Eliwa, Naglaa. Y.; Rezk-Allah, B.B. and ElShamy, M.A. (2009). Effect of organic and biofertilizer treatments on growth, flowering, bulbs production and chemical constituents of Iris tingitana cv. Wedgewood. J. Biol. Chems. Environ. Sci., 4:441-461.

El-Sayed, A.; El-Hanafy, H.; Nabih, A. and Atowa, D.I. (2012). Raising Freesia refracta cv. Red Lion corms from cormels in response to different growing media and actosol levels. J. of Hort. Sci., Ornamental Plants, 4(1):89-97.

Gangadharan, G.D. and Gopinath, G. (2000). Effect of organic and inorganic fertilizers on growth, flowering and quality of Gladiolus cv. White Prosperity. Karnataka J. Agric. Sci., 13(2):401-405 (C.F. WWW.Bids.Ac.UK).

Khan, W.; Prithiviraj, B. and Smith, D.L. (2003). Photosynthetic response of corn and soybean to foliar application of salicylates. J. Plant Physiol., 160:485492.

Moran, R. (1982). Formula determination of chlorophylls pigments extracted with $\mathrm{N}$ dimethyl-formamide. Plant Physiol., 69:1376-1381.

MSTAT-C (1986). A Microcomputer Program for the Design Management and Analysis of Agronomic Research Experiments (Version 4.0), Michigan State Univ., U.S.A.

Padmalatha, T.; Reddy, G.S.; Chandrasekhar, R.; Siva shankar, A. and Chaturvedi, A. (2014). Effect of pre- planting soaking of corms with chemicals and plant growth regulators on dormancy breaking and corm and cormel production in Gladiolus. IJPAES, 3(1):29-33.

Rajaei, N. and Onsinejad, R. (2014). Effect of municipal solid waste compost and gibberellic acid on morphological and physiological traits of tulip (Tulipa spp.) cv. Bright Parrot. European Journal of Experimental Biology, 4(1):361-368.

Ruppenthal, V. and Castro, A.M.C. (2005). Effect of urban waste compost on nutrition and yield of Gladiolus. R. Bras. Ci. Solo., 29:145-150.

Sajjad, Y.; Jaskani, M.; Ashraf, M.; Qasim, M. and Ahmad, R. (2014). Response of morphological and physiological growth attributes to foliar application of plant growth regulators in Gladiolus "White Prosperity". Pak. J. Agric. Sci., 51(1):123-129.

Sönmez, F.; Cig, A.; Gülser, F. and Basdogan, G. (2013). The effects of some organic fertilizers on nutrient contents in hybrid Gladiolus. Eurasian Journal of Soil Science 2:140-144.

Wilde, S.A.; Covey, R.P.; Lyer, J.C. and Voigt, G.K. (1985). Soil and Plant Analysis for Tree Culture. Oxford, IBH. Publishing Co., New Delhi, India.

Zaghloul, M.A.R. and Moghazy, E.I. (2001). Response of some gladiolus cultivars to organic manure and NPK fertilizers in sandy soil under Sinai conditions. Ann. Agric. Sci., Moshtohor, 39(1):585-602. 


\section{M.A.H. Abdou et al.}

\section{تأثير معاملات الكمبوست وحامضي الساليسيلك والأسكوربيك على إنتاجية الكورمات والمحتوى

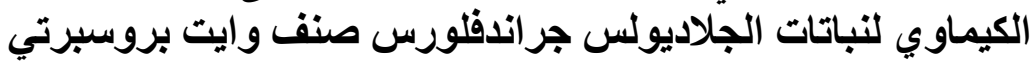

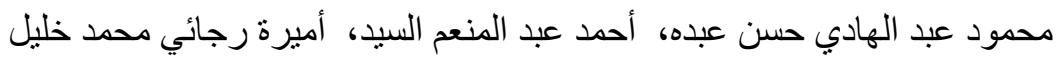

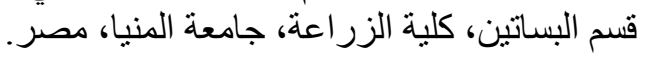

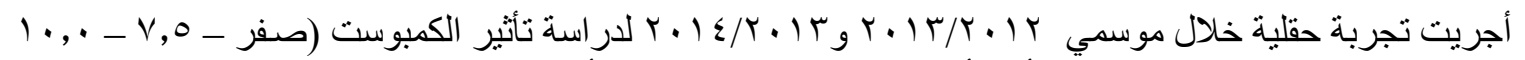

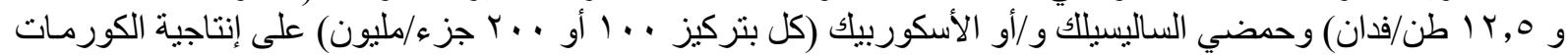

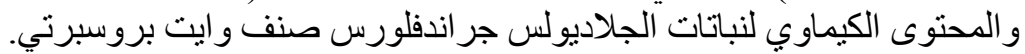

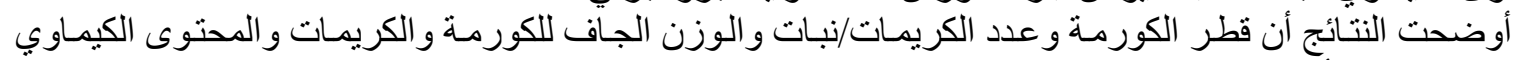

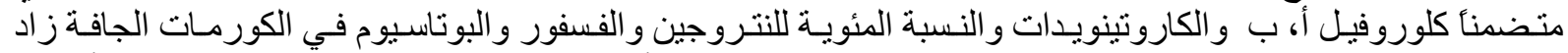

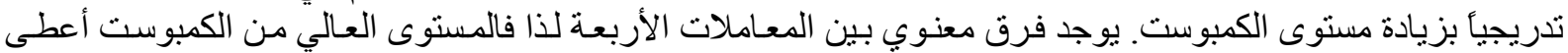
كل المعاملات الست لحمض الساليسيلك وحمض الأسكوربيك أحدثت زيادة معنوية في إنتاجية الكورمات الكمات و الكريمات

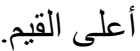

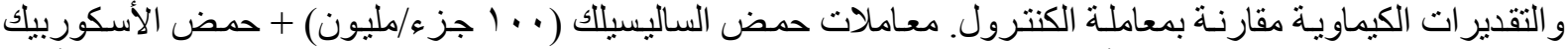

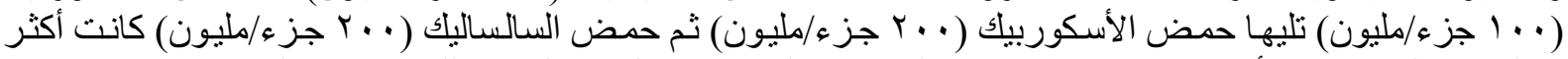

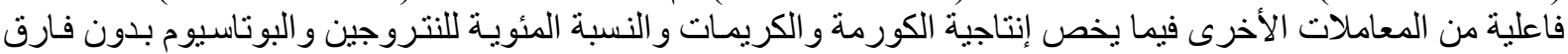

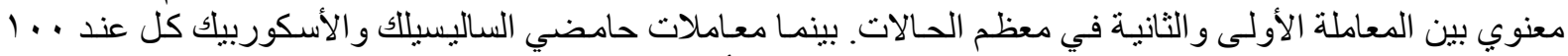

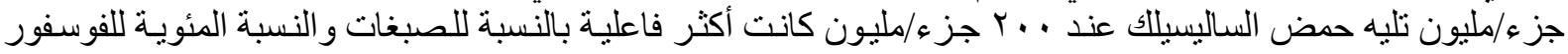
بدون فارق معنوي بينهما.

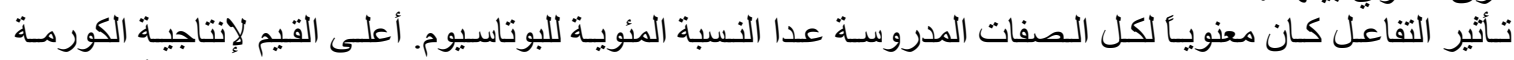

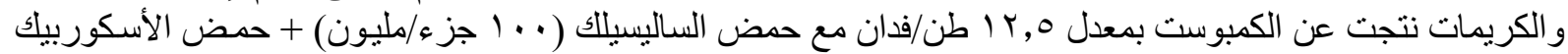

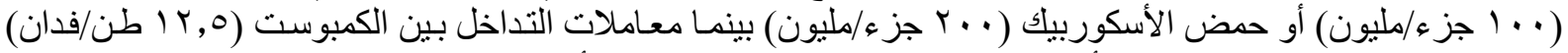

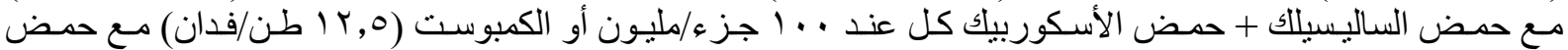

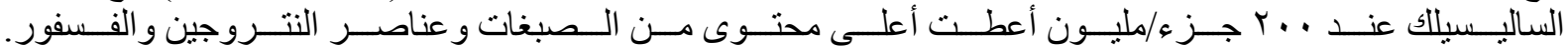

